\title{
AUTOMATIC RECOGNITION OF LEAVES BY SHAPE DETECTION PRE-PROCESSING WITH ICA
}

\author{
Jordi Solé-Casals \\ Signal Processign Group,University of Vic, Sagrada Família 7, E-08500, Vic, Spain \\ jordi.sole@uvic.cat \\ Carlos M. Travieso, Miguel A. Ferrer, Jesús B. Alonso \\ Department of Signals and Communications, Technological Centre for Innovation on Communication (CeTIC), University \\ of Las Palmas de Gran Canaria, Campus de Universitario Tafira s/n, E-35017, Las Palmas de Gran Canaria, Spain \\ \{ctravieso,mferrer,jalonso\}@dsc.ulpgc.es \\ Juan Carlos Briceño \\ Computer Science Department. University of Costa Rica \\ Sede "Rodrigo Facio Brenes", Montes de Oca, Post-Code 2060, San José. COSTA RICA. \\ juancarlos.briceno@ecci.ucr.ac.cr
}

\begin{abstract}
Keywords: Independent Component Analysis, Pattern Recognition, Leaves Recognition, Parameterization, Artificial
\end{abstract} Neural Networks.

\begin{abstract}
In this work we present a simulation of a recognition process with perimeter characterization of a simple plant leaves as a unique discriminating parameter. Data coding allowing for independence of leaves size and orientation may penalize performance recognition for some varieties. Border description sequences are then used to characterize the leaves. Independent Component Analysis (ICA) is then applied in order to study which is the best number of components to be considered for the classification task, implemented by means of an Artificial Neural Network (ANN). Obtained results with ICA as a pre-processing tool are satisfactory, and compared with some references our system improves the recognition success up to $80.8 \%$ depending on the number of considered independent components.
\end{abstract}

\section{INTRODUCTION}

Recognition of tree varieties using samples of leaves, in spite of its biological accuracy limitations, is a simple and effective method of taxonomy ( $\mathrm{Lu} e t$ al., 1994). Laurisilva Canariensis is a relatively isolated tree species, in the Canary Islands, biologically well studied and characterized. Twentytwo varieties are present in the archipelago and have simple and composed regular leaves. Our study takes into account sixteen of the twenty-two simple leaf varieties, with totals of seventy-five individuals per each one. They have been picked over different islands, pressed (for conservation purposes) and scanned in gray tonalities.
From a biological perspective, attention has to be brought to the fact that emphasis on structural characteristics, which are consistent among individuals of a species, instead of quality parameterization (as colour, size or tonality), improves recognition performance.

Quality parameterization lack of accuracy is due to the fact of leaves individual variability on the same variety as well on leaf variability on a single plant. Plant age, light, humidity, context behaviour or distribution of soil characteristics, among other things, contributes for such anomaly.

In spite of the fact that we may consider several biological parameters, as we have done previously (Loncaric, 1998), in order to generalize such study, in this paper we have just considered a border 
parameterization. This system was classified by Hidden Markov Model (HMM) (Rabiner et al., 1998) achieving a success of $78.33 \%$ (Briceño et al., 2002), and by SVM (Burges, 1998) with Principal Component Analysis (PCA) (Jolliffe, 2002) as preprocessing, achieving a success of $90.54 \%$. (SoléCasals et al., 2008)

In this present work, we have improved previous studies using the transformation and reduction of border parameterization using Independent Component Analysis (ICA) (Jutten et al., 1991) (Hyvärinen et al., 2001) and classifying its result with a Multilayer Perceptron Neural Network (MLP) (Duda et al., 2000) (Bishop, 1996).

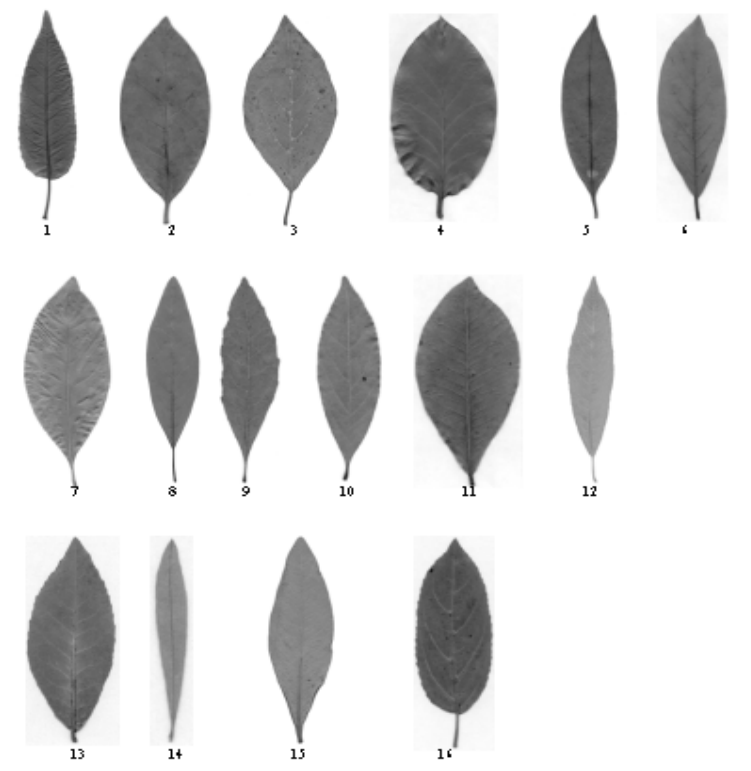

Figure 1: Images of the 16 varieties of canariensis laurisilva considered for the present study. Images are presented regardless of size.

\section{LEAVES DATABASE}

In order to create a recognition system of different vegetable species it is necessary to build a database. This database should contain the samples of the different species of study. The number of samples will be large enough to, first train the classifier with guarantees and second, test this classifier to assess the results obtained. On top of this, the amount of chosen samples, for each vegetable species must cover the largest amount of shapes and structures that this unique specie can take. In this way, a robust study of the different vegetable species is ensured.
Attending to this reasoning, the sample collection was made at different times of the year, trying in this way to cover all the colours and shapes that the leaves take throughout the four seasons. Besides, a special attention was made to reject those samples that were degraded so that the selected samples were in good condition.

Therefore, this database is composed of 16 classes (see Figure 1), with 75 samples each one. The images that form the database has been stored in a grey scale using a "jpeg" format (Joint Photographic Experts Group) with Huffman compression. The images have been digitalized to 300 dpi, with 8 bit accuracy.

\section{PARAMETERIZATION SYSTEM}

We have considered just the leaf perimeter. This image is considered without its petiole that has been extracted automatically from the shadow image. Leaves are scanned fixed on white paper sheets, placed more or less on the center, upward (petiole down) and reverse side to scan.

Border determination as $(x, y)$ positioning perimeter pixels of black intensity, has been achieved by processes of shadowing (black shape over white background), filtering of isolated points, and perimeter point to point continuous follow.

\subsection{Perimeter interpolation.}

As shown in table 1, perimeter size variability induces us to consider a convenient perimeter point interpolation, in order to standardize perimeter vector description. For an interpolating process, in order to achieve reconstruction of the original shape, we may use any of the well known algorithms as mentioned in (Lu et al., 1994), (Loncaric, 1998), (Huang et al., 1996), but a simple control point's choice criterion in 1-D analysis allows for an appropriate performance ratio on uniform control point's number and approximation error for all individuals of all varieties studied.

The general idea, for such choice, is to consider $(x, y)$ positional perimeter points as $(x, F(x))$ graph points of a 1-D relation $\mathrm{F}$.

Consideration of y coordinate as $y=F(x)$ is done, because of the way, leaves images are presented in our study: leaves have been scanned with maximum size placed over $x$ ordinate. 
Table 1: A comparative table of mean error, obtained from a uniform criterion of control point selection and the monotonic way

\begin{tabular}{cccc}
\hline \multirow{2}{*}{ Class } & Mean size & \multicolumn{2}{c}{ Mean Error } \\
& & Uniform & Monotonic \\
\hline 01 & 2665.6 & 9.1474 & 2.0226 \\
02 & 1885.1067 & 3.5651 & 0.43655 \\
03 & 2657.68 & 11.0432 & 5.3732 \\
04 & 2845.8133 & 31.6506 & 2.8447 \\
05 & 1994.68 & 1.8569 & 0.42231 \\
06 & 2483.04 & 0.4425 & 0.71093 \\
07 & 2365.2667 & 9.711 & 0.68609 \\
08 & 3265.48 & 0.4753 & 0.49015 \\
09 & 2033.2267 & 19.7583 & 3.4516 \\
10 & 2258.2533 & 3.9345 & 2.4034 \\
11 & 1158.9867 & 5.4739 & 1.0286 \\
12 & 1934 & 1.3393 & 0.40771 \\
13 & 1183.4 & 1.2064 & 0.39012 \\
14 & 981.4 & 0.2752 & 0.23671 \\
15 & 3159.08 & 11.575 & 8.8491 \\
16 & 1973.3733 & 47.4766 & 6.6833 \\
\hline
\end{tabular}

For a relation $G$ to be considered as a onedimensional function, there is need to preserver a correct sequencing definition (monotonic behaviour). That is: A graph

$$
G=\left\{i=1 . . n,\left(x_{i}, y_{i}\right) / y_{i}=f\left(x_{i}\right)\right\}
$$

It is the description of a function $\mathrm{f}$ if ordinate points $x_{i}, i=1 . . n$ must be such that: $x_{i}<x_{i+1}, i=1 . . n-1$. We consider then the border relation $\mathrm{F}$ as a union of piece like curves (graphs) preserving the monotonic behaviour criterion, i.e.

$$
F=\bigcup_{j \in J} G_{j}
$$

where $G_{j} \subseteq F, \forall j \in J$ and

$$
G_{j}=\left\{\boldsymbol{\alpha}_{j} \in J_{j},\left(x_{\boldsymbol{\alpha}_{j}}, y_{\boldsymbol{\alpha}_{j}}\right) / y_{\boldsymbol{\alpha}_{j}}=f_{j}\right\}
$$

For convenient sets of index $\mathrm{J}, \mathrm{J}_{\mathrm{j}}$ and restriction functions $f_{j}=f_{\left\{\left\{x_{\boldsymbol{\alpha}_{j}} \mid \boldsymbol{\alpha}_{i} \in J_{j}\right\}\right.}$, such that the next point following the last of $G_{j}$ is the first one of $G_{j+l}$. $G_{j}$ graphs are correct $f_{j}$ functions descriptions.

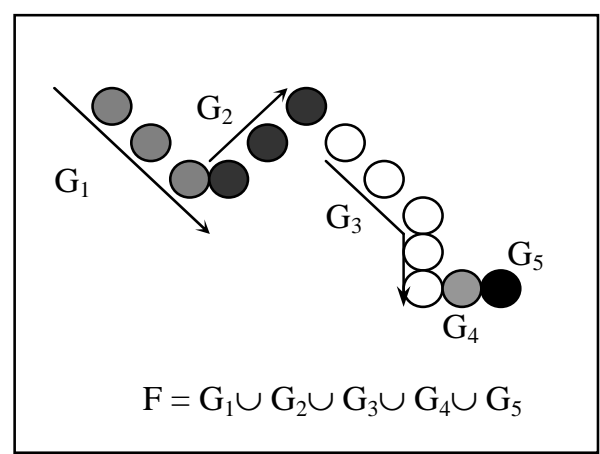

Figure 2: Example of an $\mathrm{F}$ relation decomposed in graphs with a correct function description.

Building the $G_{j}$ sets is a very straightforward operation:

- Beginning with a first point we include the next one of $F$.

- As soon as this point doesn't preserve monotonic behaviour we begin with a new $G_{j+1}$.

- Processes stop when all $F$ points are assigned.

In order to avoid building $G_{j}$ reduced to singletons, as show in figure $2\left(G_{4}\right.$ and $\left.G_{5}\right)$ the original $F$ relation may be simplified to preserve only the first point of constant $\mathrm{x}$ ordinate series. 
Afterwards, spreading of a constant number of points is done proportional to the length of the $G_{j}$ and always setting in it is first one.

The point's choice criterion mentioned before allows, in two-dimensional interpolation, for taking account on points where reverse direction changes take place. Irregularity, of the surface curve, is taken into account with a sufficient number of interpolating points, as done in the uniform spreading way. Results on table 1 allows for comparison between choice of control points with the criterion motioned before and the uniform one. Such results show the benefit of choosing control points with the monotonic criterion instead of the uniform one.

The 1-D interpolation has been perform using 359 control points, with spline, lineal or closest interpolated point neighbourhood, depending on the number of control points present in the decomposed curve. As a reference at 300 dpi a crayon free hand trace is about 5 to 6 points wide.

Table 1 also shows size variability of the different varieties ranging in mean, between 981 pixels for class 14 to 3255 for class 8 . With 359 points chosen with the monotonic criterion, all perimeter point vectors have a standard size and errors representation is negligible.

Due to perimeter size variability inside a class, for example in class 15 ranging between 2115 points to 4276 with a standard deviation of about 521 , coding of $(x, y)$ control perimeter points have been transformed taking account for size independence.

Considering the following definitions:

$\Gamma$ the set of $\mathrm{n}$, a fixed number, of control points, $\Gamma=\left\{X_{i=1 . . n} / X_{i}=\left(x_{i}, y_{i}\right)\right\}$ Where $\left(x_{i}, y_{i}\right)$ are point coordinates of control perimeter points.

$\mathrm{C}_{0}$ the central point of the $\Gamma$ set: $C_{0}=(1 / n)\left(\sum_{i=1 . . n} x_{i}, \sum_{i=1 . . n} y_{i}\right)$,

$/\left(x_{i}, y_{i}\right)_{i=1 . . n} \in \Gamma$,

$\boldsymbol{\beta}_{i}=\operatorname{angle}\left(C_{0} X_{i} X_{i+1}\right), \boldsymbol{\alpha}_{i}=\operatorname{angle}\left(X_{i} C_{0} X_{i+1}\right) \quad$ angles defined for each interpolating points of $\Gamma$.

An example is shown in figure 3 . Sequences of $\left(x_{i}, y_{i}\right)$ positional points are then transformed in sequence of $\left(\varphi_{i}, \beta_{i}\right)$ angular points.

The choice of a starting and a central point accounts for scale and leaf orientation. Placement of both points sets the scale: its distance separation. Relative point positioning sets the orientation of the interpolating shape. Given a sequence of such angles $\alpha_{i}$ and $\beta_{i}$, it's then possible to reconstruct the interpolating shape of a leaf. Geometrical properties of triangle similarities make such sequence size and orientation free.

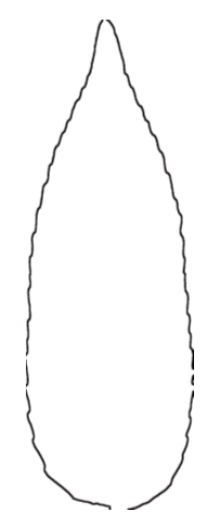

Example of shape representation

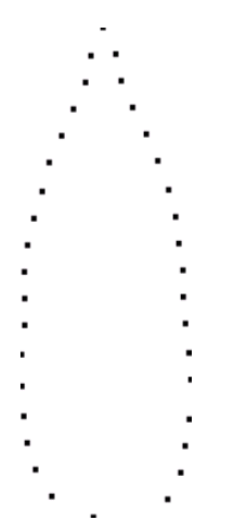

$\left(x_{i}, y_{i}\right)$ sequence of control point choice selection

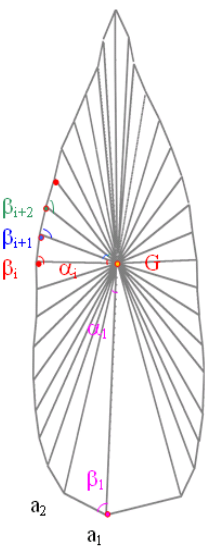

$\left(\alpha_{i}, \beta_{i}\right)$ sequence transform
Figure 3: Example of an angular coding for a 30 control points selection.

\section{REDUCTION PARAMETERS}

The problem of classification consists on deciding a class membership of an observation vector (Duda et al., 2000). Usually this observation vector consists of features that are related. The classification algorithm has to take a decision after the analysis of several features even though they can be mutually related in difficult ways.

The dependencies between the features have an influence on the learned classifier. It is well known that there is a relationship between the complexity of a classifier and the generalization error (Mitchell, 1997).

We propose transforming the input so that the resulting vector has the property that each component is independent of the others. We shall do this by means of ICA. In the case of training a multilayer perceptron, the inference of the weights is made by a gradient search, which is known to be very inefficient if the features are highly correlated (Duda et al., 2000). It is also known that the incorrelation pre-processing of the inputs of a multilayer perceptron improves the convergence of the algorithm because near a minimum the form of the error function can be approximated locally by a hyper-parabola. This explains the improvement that can be achieved by the use of algorithms such 
as the conjugate gradient or the LevenbergMarquardt.

Notice that the characteristics of these algorithms are adapted to the fact that the data can have correlated features. So a process of whitening the data or using these improvements of the gradient algorithms means that we are making a strong hypothesis about the data.

In the past, ICA has been used as a preprocessing technique for classification (SanchezPoblador et al., 2004), where the authors proposed the use of the independent component analysis technique for improving the classification rate of decision trees and multilayer perceptrons. We propose to follow the same idea to pre-process the data in such a way that the features will be mutually independent, and therefore the gradient descent will follow a smooth surface, even if high order moments between features are present in the original pattern.

See (Jutten et al., 1991) (Hyvärinen et al., 2001) for a detailed explanation of ICA theory and algorithms. In all the experiments the ICA transformation was done by means of the JADE algorithm (Cardoso et al., 1996).

\section{CLASSIFICATION}

An Artificial Neural Network (ANN) can be defined as a distributed structure of parallel processing, formed by artificial neurons, interconnected by a great number of connections (synapses) (see Figure 4).

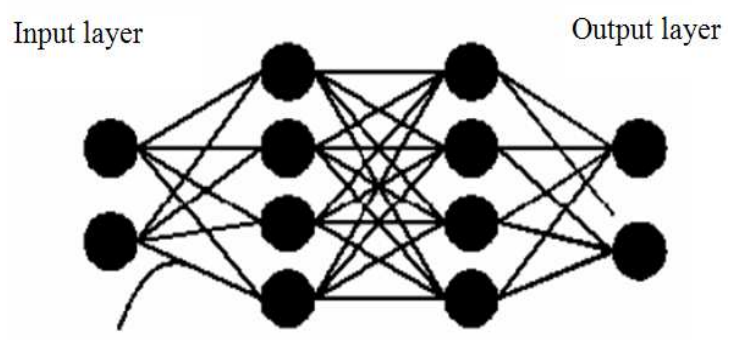

Connections

Figure 4: General Neural Network structure

Feed-Forward networks consist of layers of neurons where the exit of a neuron of a layer feeds all the neurons on the following layer. The fundamental aspect of this structure is that feedback unions do not exist. The so called
Multilayer Perceptron (MLP) is a type of Feedforward ANN, where the threshold function is a nonlinear but differentiable function. This nonlinear differentiable function is necessary to assure that the gradient can be calculated, in order to find the good values for all the parameters of the network.

For the classification system we have used a 2layer feed-forward perceptron trained by means of conjugated gradient descent algorithm (Bishop, 1996), with 50 neurons in the hidden layer and hyperbolic tangent $\tanh ($.$) as a nonlinear function$ for these units. The number of input neurons fits in with the number of components (from 1 to 15 ), and the number of output neurons with the number of leaves classes (16 in our application).

Approximately half of the database (37) was used in the training process and the rest of the examples (38) for testing the network.

\section{EXPERIMENTS AND RESULTS}

We did several experiments in order to find the best dimension reduction for leaves automatic recognition. In our experiments we observed that the first column of each sample, that corresponds to the interior angles $\alpha_{i}$ are not useful for the classification purpose. Hence, we use only exterior angles $\beta_{i}$.

To apply ICA to these values we construct a global matrix with 37 of 75 different samples that we have for each class, arranged in rows, resulting in a 592x359 global matrix. ICA algorithm, as detailed in Section 4, is then applied to this matrix in order to obtain the projected data by using the subspace spanned from 5 to 15 independent components (only odd numbers in our experiment).

Data processed with ICA is used then with a neural network and results are shown in Table 2 . For each number of components we trained 10 different networks and we calculated the success of the classification procedure, showing the success rate and standard devition value for each case.

We obtained better results compared with these obtained in (Briceño et al., 2002) where a HMM of 40 stages was used in the best case, giving a success rate of $78.33 \% \pm 6.06$.

With the new ICA pre-processing procedure we outperform previous results in success rate and we diminish the variance as well. With 15 independent components we obtain $80.77 \% \pm 0.29$ of success, in the classification of leaves for 10 different trained neural networks. The variance in the 
classification is strongly diminished from 6.06 for the HMM (Briceño et al., 2002) to less than 0.80 in all the cases.

Also is interesting to observe that with 13 independent components we obtain a very good result of $80.40 \% \pm 0.78$ that outperforms the previous results showed in (Briceño et al., 2002). As the number of components is small, we simplify the classification step.

Table 2: Results with MLP classifier

\begin{tabular}{cc}
\hline $\begin{array}{c}\text { Number of } \\
\text { Component }\end{array}$ & Success rate \\
\hline ICA 5 & $74.93 \% \pm 0.75$ \\
ICA 7 & $75.76 \% \pm 0.62$ \\
ICA 9 & $78.01 \% \pm 0.55$ \\
ICA 11 & $79.10 \% \pm 0.51$ \\
ICA 13 & $80.40 \% \pm 0.78$ \\
ICA 15 & $80.77 \% \pm 0.29$
\end{tabular}

\section{CONCLUSIONS}

In this present work, we have presented an improvement of an automatic leaves recognition system using Independent Component Analysis and classifying with multilayer perceptron neural network. The transformation and reduction of data contribute to increase its discrimination, from $78.33 \%$ using contour parameterization + HMM (Briceño et al., 2002), to $80.77 \%$ using contour parameterization + ICA + Neural Network.

The advantage of using ICA is twofold: first, we increase the classification results, specially diminishing the variance, and second we reduce the features dimension, giving as a result a less complex classifier.

Future work will be done exploring other different ICA algorithms combined with other classifiers in order to diminish the complexity of the whole classification system.

\section{ACKNOWLEDGEMENTS}

The first author acknowledges support from the Ministerio de Educación y Ciencia of Spain under the grant TEC2007-61535/TCM, and from the Universitat de Vic under the grant R0912.

\section{REFERENCES}

Lu, F., Milios, E. E., 1994. Optimal Spline Fitting to Planar Shape. In Elsevier Signal Processing, No. 37, pp 129-140.

Loncaric, S., 1998. A Survey of Shape Analysis Techniques. In Pattern Recognition, Vol. 31 No. 8, pp 983-1001.

Rabiner, L., Juang, B H., 1993. Fundamentals of Speech Recognition. Prentice Hall, Englewood Cliffs, New Jersey.

Briceño, J. C., Travieso, C. M., Ferrer, M. A., 2002. Automatic Recognition of Simple Laurisilva Canariensis Leaves, by Perimeter Characterization. In IASTED International Conference on Signal Processing, Pattern Recognition and its Applications, pp. 249 - 254.

Burges, C.J.C., 1998. A Tutorial on Support Vector Machines for Pattern Recognition. In Data Mining and Knowledge Discovery, Vol. 2, N², pp. 121-167.

Jolliffe I.T., 2002. Principal Component Analysis. Series: Springer Series in Statistics, Springer, NY 2nd edition.

Jutten, C., Herault, J., 1991. Blind separation of sources, Part 1: an adaptive algorithm based on neuromimetic architecture. In Signal Processing (Elsevier), Vol. 24, Issue 1, pp. 1-10

Hyvärinen, A., Karhunen, J., Oja, E., 2001. Independent Component Analysis, New York, USA: John Wiley $\&$ Sons.

Duda, R.O., Hart, P.E., Stork, D.G., 2000. Pattern Classification. Wiley Interscience, 2nd Edition.

Bishop, C., 1996. Neural Networks for Pattern Recognition. Clarendon, UK: Oxford University Press.

Lu, F., Milios, E.E., 1994. Optimal Spline Fitting to Planar Shape. In Elsevier Signal Processing No. 37, pp. 129-140.

Loncaric, S., 1998. A Survey of Shape Analysis Techniques. In Pattern Recognition. Vol. 31 No. 8, pp. 983-1001.

Huang, Z., Cohen, F., 1996. Affine-Invariant B-Spline Moments for Curve Matching. In IEEE Transactions on Image Processing, Vol. 5. No. 10. pp. 824-836.

Mitchell, T.M., 1997. Machine Learning. McGraw-Hill.

Sanchez-Poblador, V., Monte-Moreno, E., Solé-Casals, J., 2004. ICA as a preprocessing technique for Classification. In Independent Component Analysis and Blind Signal Separation, Lecture Notes in Computer Science, Springer-Verlag Volume 3195/2004, pp. 1165-1172.

Cardoso, J.F., Souloumiac, A., 1996. Jacobi angles for simultaneous diagona-lization, In SIAM. Journal Mathematics Analysis Application, pp. 161-164. 